\title{
Research on patients-passive control strategy of the rehabilitation exoskeleton based on NN-SMC
}

\author{
C Chen ${ }^{\mathrm{a}}$, S M Zhang, Z Y Xu, and J Y Shen \\ College of Mechanical and Transportation Engineering, China University of Petroleum, Beijing, China
}

\begin{abstract}
There are amounts of patients with locomotor dysfunction caused by stroke until now. Body weight supported treadmill training (BWSTT) has proved to be an efficient method of rehabilitation training for those people. The lower exoskeleton consists of two legs which is used to guide and assist motions of patients with the help of weight support devices and a treadmill. A prototype of the body weight support exoskeleton rehabilitation device (BWSERD) has been designed in this paper, which contains two pairs of direct drives at hip and knee joints. It has also four torque transducers and four encoders. In order to conduct the patients-passive rehabilitation training after stroke, a control strategy based on neuro network and sliding mode controller is developed. The effectiveness of the proposed method is confirmed by the simulation results.
\end{abstract}

\section{Introduction}

Stroke is a kind of cardiovascular and cerebrovascular disease. There are amounts of patients with locomotor dysfunction caused by stroke until now. According to theory of central nervous system plasticity, the appropriate regular training contributes to the rehabilitation of motor ability for patients. Body weight supported treadmill training (BWSTT) is a general rehabilitation training method for humans with locomotor dysfunction at present.

Considering that the disadvantages exist in BWSTT assisted by humans, BWSTT associated with robotic devices is proposed to gain a better training performance. The rehabilitation robot LOKOMAT developed at the Spinal Cord Injury Center of the University Hospital Balgrist is one of the most popular rehabilitation robots in the world. In clinical use, LOKOMAT takes advantage of the position-controlled algorithm to train patients in a passive way [1]. Passive training ignores the muscle activity of patients. In order to maximize the participation of patients, three algorithms adapting to the gait patterns of patients are proposed in LOKOMAT [2]. Besides, an impedance control strategy in LOKOMAT has been designed, which takes into account the patient's intention and voluntary efforts by an adjustable virtual springdamper element [3]. This strategy allows the patients to deviate from the reference gait pattern. An adaptive control method is demonstrated to help the legs move in a desired periodic trajectory which incorporates the learning approaches in order to tackle periodic uncertainties with known periods [4]. Exoskeleton and functional electrical stimulation (FES) approach is used for rehabilitation training of individuals with spinal cord injury, which incorporates the advantages of both methods and eliminates the disadvantages [5]. An algorithm is proposed in Robot Suit HAL designed by University of Tsukuba to estimate patients' intentions so that HAL could comfortably and safely support a paraplegia patient's walking [6]. Furthermore, HAL takes the principle component analysis method to evaluate the working performance, which demonstrates the improvement of patients' gait coordination [7]. Brainmachine interfaces (BMIs) system is reviewed, which concludes that BMIs open the new possibilities in the assistance and rehabilitation fields [8]. A lower extremity exoskeleton named CUHK-EXO was developed, which can help patients to conduct stand up/sit down and walking training [9]. A co-simulation research of exoskeleton-human robot system with fuzzy-PID/PID algorithms is conducted, and the performance is verified in five scenarios such as level walking, stair ascent, stair descent and so on [10].

A prototype of the body weight support exoskeleton rehabilitation device (BWSERD) has been designed in this paper. With the help of this device, patients can get extended time to train effectively. At the same time, it only needs a therapist who can save more energy. Above all, the gait pattern for training is more accurate, which can be guaranteed by the control strategy based on neuro network and sliding mode controller. This paper is organized as follows. Firstly, the mechanical structure and dynamic models of BWSERD are described. Thereafter the human physiological gait data is introduced, which is used as the reference gait trajectory of training. And then, sliding mode controller combined with neuro networks is designed. Next, the simulation research is demonstrated. Finally, the conclusion and future work are given.

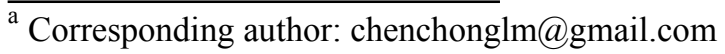




\section{Mechanical structure dynamic models}

\subsection{Design of BWSERD}

The BWSERD consists of four direct drives at both hips and knees as is showed in figure 1. The support aluminium alloy skeleton outside is omitted in order that this device is demonstrated clearly. The exoskeleton only remains. Each hip and knee is both rotational degrees of freedom, which is driven directly by a servo motor combined with a harmonic reducer. A torque transducer and a coder are equipped at every hip and knee joint. The ankles are also rotational degrees of freedom, which are passive. The ankle is guided by the treadmill during stance phases and is provided only a little torque by patients during swing phases.

The exoskeleton is fixed on two sliding rails which are attached on the aluminium alloy skeleton outside. The exoskeleton is limited to move only in the vertical direction. There are two cuffs on each thigh and shank of the exoskeleton, which guide legs of a patient to move in concert with legs of the exoskeleton. What's more, the length of each thigh and shank is adjustable easily, which makes it suited to people of different shapes.

\section{Sliding rail}

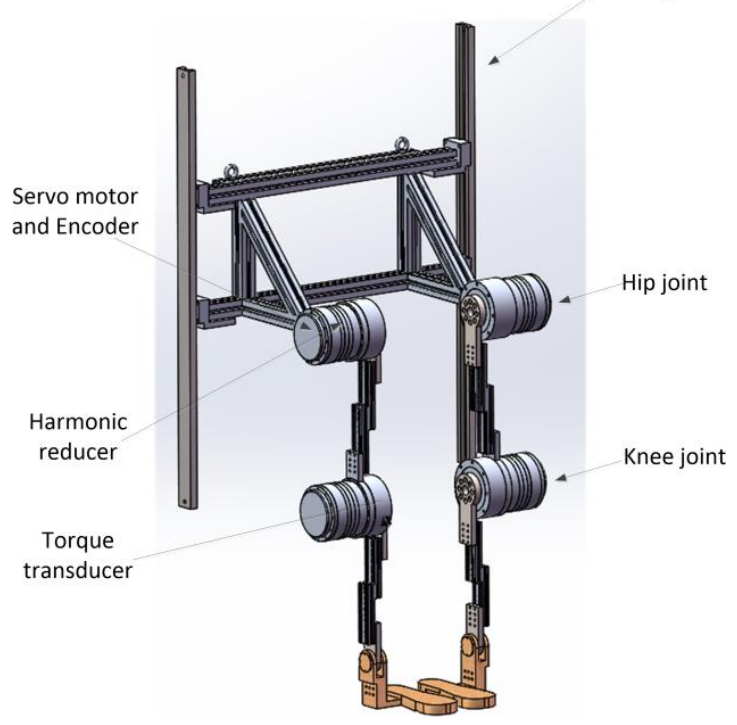

Figure 1. Mechanical structure of the exoskeleton.

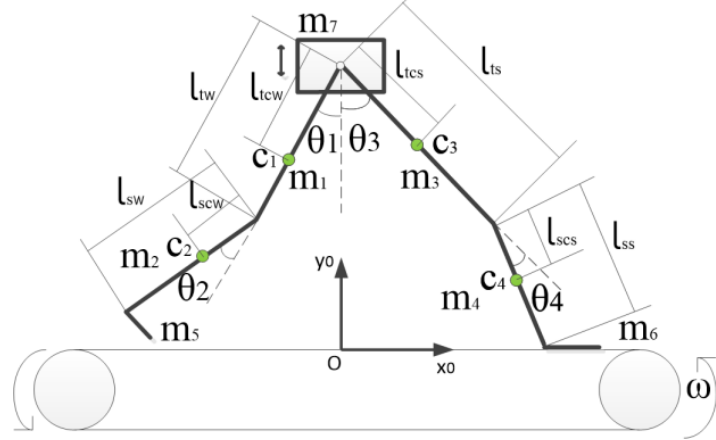

Figure 2. Dynamic model of exoskeleton.
Each hip and knee of the exoskeleton is directly driven by a motor and a harmonic reducer. As a result, the dynamic model of drive unit can be described as equation (1).

$J \ddot{\theta}_{m}+B \dot{\theta}_{m}=T_{m}-T_{l}^{\prime}$

Where $\theta_{m}$ is the angular displacement of the servo motor. $J$ is the moment of inertia on the motor shaft. $B$ is the damping coefficient. $T_{m}$ is the output torque of the motor. $T_{l}^{\prime}$ equals $T_{l}$ divided by $R . T_{l}$ is the joint torque. $R$ is the reduction ratio of the harmonic reducer.

\subsection{Dynamic model of exoskeleton.}

Generally, the gait period of human could be divided into the single-support phase and the double-support phase to establish dynamic models respectively [11]. Considering that the patient's hip and knee joints cannot provide enough joint torque for normal walking, active driving is only used at the hip and knee joints while the ankle joint is passive. The elastic energy storage unit at the ankle joint makes the patient's foot plate almost parallel to the treadmill throughout the rehabilitation process. According to kinematics simulation, the duration of double-support phase is much shorter than the single-support phase under that condition. Therefore, when establishing the dynamic model, only single-support phase is considered. The dynamic model of exoskeleton is established as is shown in figure 2. Each center of mass for legs of exoskeleton can be gotten in reference coordinate $X \mathrm{o}-\mathrm{O}-Y_{\mathrm{o}}$. The Lagrangian method is used for gaining the dynamic equations of the exoskeleton. Lagrangian function is described as equation (2).

$L=\frac{1}{2} \sum_{i=1}^{4}\left(m_{i} v_{c i}^{2}+J_{i} \theta_{i}^{2}\right)+\frac{1}{2} \sum_{i=5}^{8}\left(m_{i} v_{i}^{2}\right)-\sum_{i=5}^{8}\left(m_{i} g y_{i}\right)$

Where $m_{i}$ is the mass of each thigh or shank. $J_{i}$ is the moment of inertia of each thigh or shank. $\theta_{i}$ is the angular displacement of each thigh or shank. $y_{i}$ is the height of center of mass. From equation (2), Lagrangian equation is described as equation (3). By solving equation (3), the torque of each joint can be acquired.

$\left[\frac{d}{d t}\left(\frac{\partial L}{\partial \dot{q}_{i}}\right)-\frac{\partial L}{\partial q_{i}}\right]=\left[T_{i}\right](i=1,2,3,4)$

\section{Clinical gait data}

The clinical gait data used in this paper is derived directly from the Clinical Gait Analysis (CGA) Normative Gait Database [12]. Clinical gait data is a series of discrete values. In fact, the movement of the human is continuous. Therefore, it is firstly necessary to fit those data based on the principle of least squares. Considering the gait characteristics of the human, curve fitting is conducted in the form of the triangular series. The function used for curve fitting is described as equation (4). Where $\theta$ is the fitted angle. $t$ is time. $n$ is order of fitting. $N$ is chosen according to precision. $A_{n}, f, \varphi, A_{0}$ can be

\subsection{Dynamic model of drive unit.}


gotten by the principle of least squares. The fitting curve can reflect the physiology gait of normal human correctly, which can be used as the reference trajectory of position control algorithm.

$\theta=\sum_{n=1}^{N} A_{n} \sin (2 n \pi f t+\varphi)+A_{0}$

\section{Design of controller}

\subsection{Sliding mode controller.}

Firstly, the sliding function is defined as equation (5).

$$
s(t)=e(t)+\lambda e(t)
$$

Where $e(t)$ and $e(t)$ denote tracking error and its derivative separately. When $s(t)$ equals zero, $e(t)+\lambda e(t)$ equals zero too. Equation (6) can be acquired.

$$
e(t)=e(0) \exp (-\lambda t)
$$

In order to make sure the system is asymptotically stable, $\lambda$ must be greater than zero. The Lyapunov function is defined as equation (7).

$v=s^{2} / 2$

Equation (8) can be acquired from equation (7).

$\dot{v}=s \dot{s}=s(\ddot{e}(t)+\lambda \dot{e(t)})=s\left(\ddot{\theta_{m}}-\ddot{\theta_{d}}+\lambda \dot{e(t)}\right)=s\left[\left(T_{m}-T_{l}^{\prime}-B\right.\right.$

According to the Lyapunov's stability theory, $s s$ must be less than zero. As a result, control law can be designed as equation (9).

$$
T_{m}=J \ddot{\theta_{m}}+B \dot{\theta_{m}}-J \lambda \dot{e(t)}-\eta \operatorname{sgn}(s)
$$

Where $\eta \geq \max \left|T_{l}^{\prime}\right|$.

Equation (9) is a standard sliding mode controller, where $\lambda$ determines the rate of convergence. In order to resist the disturbances and uncertainties of modelling, $\eta$ is generally relatively large. Huge chattering of the system will happen, which causes adverse effects to the system. The neuro network is introduced to eliminate the adverse effects.

\subsection{Neuro network-sliding mode controller (NN-SMC)}

The Radial Basis Function (RBF) neural network was proposed in 1988. The RBF neural network is capable of approximating any nonlinear function with any precision [13]. The structure of the RBF neural network is shown in figure 3. $s$ is input of the RBF neural network while $T(s)$ is the output. $T(s)$ equals $\omega^{T} \phi(s) . \omega$ is the weight vector. $\phi(s)$ is the basis function. Gaussian radial base function is used in this paper. It is defined as eqution (10). $\phi(s)_{i}=\exp \left(-\frac{\left\|s-c_{i}\right\|}{\sigma_{i}^{2}}\right)$

Where $c_{i}$ is the center of the radial basis function. $\sigma_{i}$ is the width of the radial basis function. RBF neural network is used to approximate $-\eta \operatorname{sgn}(s)$, which can combine neuro network with sliding mode controller effectively. RBF networks have self-learning capabilities that can adapt to changes in external loads and disturbances, which will weaken chattering. Equation (9) will change to equation (11).

$$
T_{m}=J \ddot{\theta_{m}}+B \dot{\theta_{m}}-J \lambda \dot{e(t)}+\omega^{T} \phi(s)
$$

To adapt to changes in loads and interferences, the weight, the center, and the width need to be adaptively adjusted.

The objective function to adjust is that $\dot{s}$ equals zero. The gradient descent method is taken to adjust these parameters as is shown in eqution (12).

$$
\begin{aligned}
& \omega(\mathrm{t}+1)=\omega(\mathrm{t})-\beta\left(\frac{\partial(\dot{\mathrm{s} s})}{\omega}\right) ; \frac{\partial(\mathrm{ss})}{\partial \omega}=\frac{\partial(\mathrm{s})}{\partial T_{m}} \frac{\partial T_{m}}{\partial \omega}=s \phi(\mathrm{s}) \\
& c(\mathrm{t}+1)=c(\mathrm{t})-\beta\left(\frac{\partial(\dot{\mathrm{s}})}{c}\right) ; \quad \frac{\partial(\dot{\mathrm{s}})}{\partial c}=\frac{\partial(\dot{\mathrm{ss}})}{\partial T_{m}} \frac{\partial T_{m}}{\partial c}=2 \omega \phi(\mathrm{s})(\mathrm{s}-\mathrm{c}) / \sigma \\
& \sigma(\mathrm{t}+1)=\sigma(\mathrm{t})-\beta\left(\frac{\partial(\dot{\mathrm{s}})}{\sigma}\right) ; \frac{\partial(\dot{\mathrm{s}})}{\partial \sigma}=\frac{\partial(\dot{\mathrm{s}})}{\partial T_{m}} \frac{\partial T_{m}}{\partial \sigma}=2 \omega \phi(\mathrm{s})\|\mathrm{s}-\mathrm{c}\|^{2} / \sigma^{3}
\end{aligned}
$$

Where $\beta$ is the adjustment size. Hidden Layer

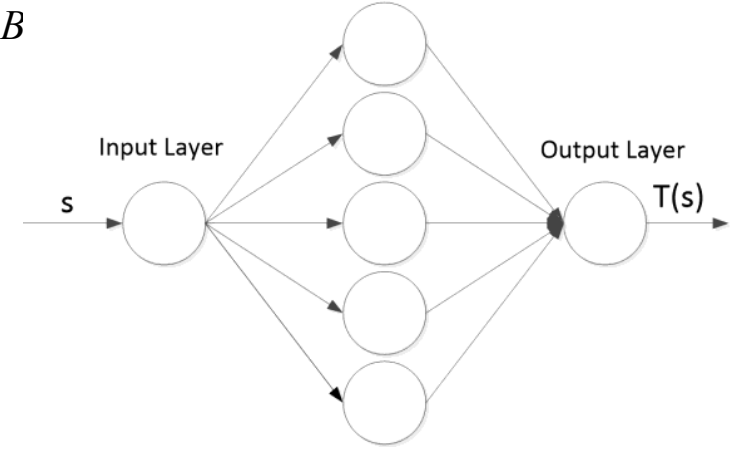

Figure 3. RBF neural network.

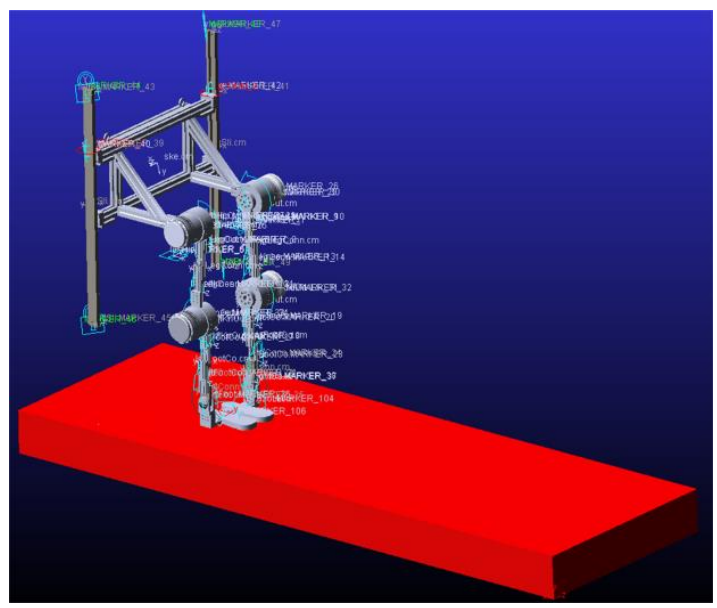

Figure 4. Dynamic model in ADAMS. 


\section{Simulation and analysis}

\subsection{Dynamic simulation of exoskeleton.}

Automatic dynamic analysis of mechanical systems (ADAMS) has powerful kinematics and dynamics analysis functions, while its modeling function is poor. This paper builds up a three-dimensional solid exoskeleton model in SolidWorks. And then the model is inputted into ADAMS for dynamic simulation analysis. The exoskeleton ADAMS dynamics model is shown in figure 4.

Since the material properties and contact characteristics are lost after the model built in SolidWorks is inputted into ADAMS, it is necessary to supplement those characteristic parameters firstly. According to the normal gait trajectory obtained in the previous section, it is imported into the ADAMS as the joint trajectory. The joint torque is obtained by simulation analysis. Considering the symmetry of the exoskeleton, only the joint torque curves of the left leg are shown in figure 5 . The curve starts from stance state of the person. Its gait period is one second. The simulation lasts 10.5 seconds. In addition to the initial step, there are a total of 9 gait cycles.
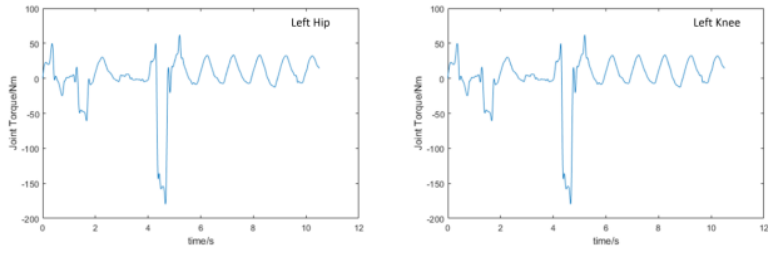

Figure 5. Torque curves of the left leg.

\subsection{Simulation of controller}

The exoskeleton is a complex time-varying, nonlinear dynamic system. For the rehabilitation training, it is necessary to force the human legs to move in concert with the joint trajectory of the exoskeleton in the passive training mode. The patient's morbidity gait, such as spastic gait, will disturb the movement of the exoskeleton according to a normal gait tracking. At the same time, resisting these disturbances plays a significant role in the patients' training according to the normal gait trajectory, which will prevent humans from learning the morbidity gait. Considering the influence of modeling uncertainties, a robust position control algorithm needs to be proposed. This paper applies the NN-SMC control algorithm. The design of the controller is shown in previous section. In simulation, $J$ equals $9.38 \mathrm{~kg} . \mathrm{cm}^{2}$. $B$ equals $3 \mathrm{~N} . \mathrm{cm} / \mathrm{krpm}$. These parameters are adapted to be the same as our experimental prototype. $\lambda$ equals 1 . Besides, a RBF neuro network with five hidden layer neuron is utilized. The simulation results based on NN-SMC is shown in figure 6 and figure 7, compared with the only sliding mode controller.
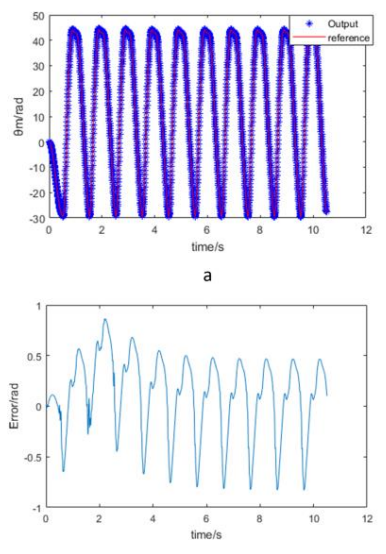

c

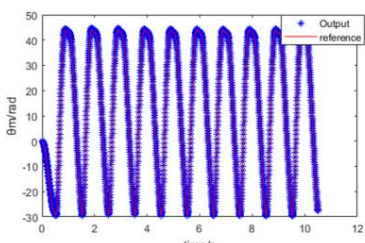

b

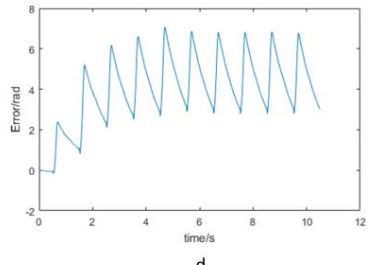

Figure 6. a) The result of trajectory tracking when NNSMC is adopted on the left hip joint. b) The result of trajectory tracking when sliding mode controller is adopted on the left hip joint. c) The error of trajectory tracking based on NN-SMC on the left hip joint. d) The error of trajectory tracking based on sliding mode controller on left the hip joint.
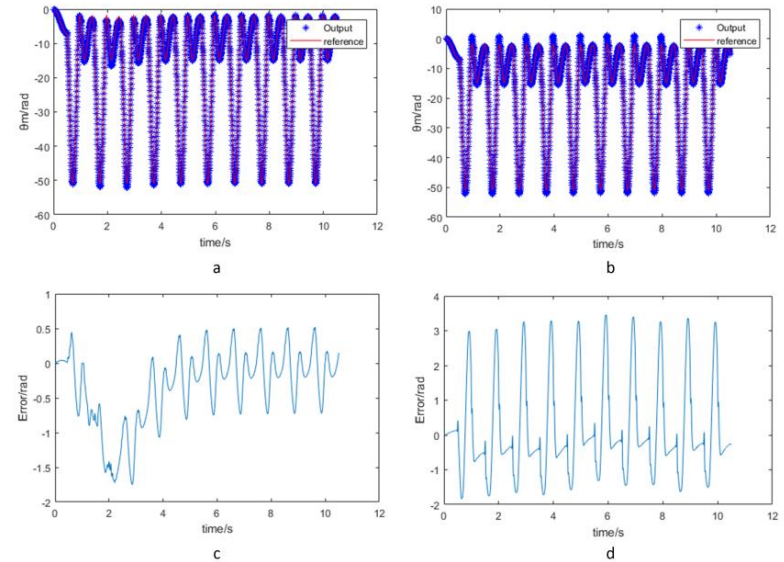

Figure 7. a) The result of trajectory tracking when NNSMC is adopted on the left knee joint. b) The result of trajectory tracking when sliding mode controller is adopted on the left knee joint. c) The error of trajectory tracking based on NN-SMC on the left knee joint. d) The error of trajectory tracking based on sliding mode controller on the knee left joint.

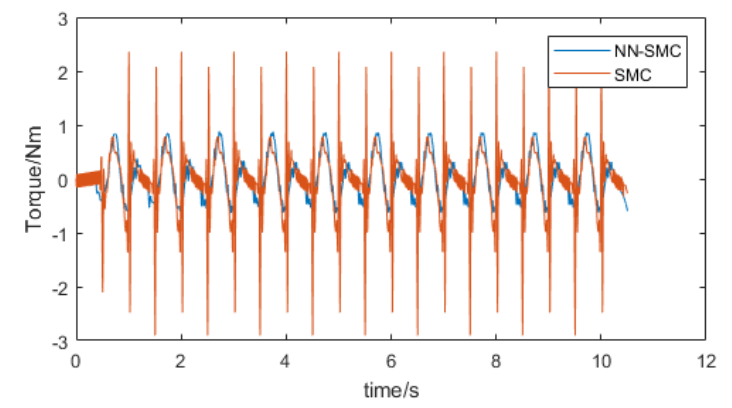

Figure 8. Output torques of different controllers

The simulation results show that the position control algorithm based on NN-SMC can effectively track the ideal gait trajectory of the normal human. The error of trajectory tracking is less than $1 \mathrm{rad}$ on the hip joint and 2 rad on the knee joint. Compared with the results based on only sliding mode controller, those errors are much 
smaller. The change rates of errors are much smaller as well. The output torques of controller on the left knee joints are shown in figure 8 .

From figure 8 , it can be concluded that application of NN-SMC alleviates the chattering of system efficiently. As a result, the NN-SMC is robust to changes in external loads and modelling uncertainties. NN-SMC is truly applicable to the control of exoskeleton when the training of patients is in a passive way.

\section{Conclusion and future work}

This paper proposes a passive training algorithm based on NN-SMC for lower extremity rehabilitation exoskeleton. This algorithm combines the advantages of neural network and sliding mode variable structure control. The neural network learns the changes of loads, which weakened the chattering of the system and eliminated the deficits of the sliding variable structure control. Besides, the errors based on NN-SMC are much smaller than ones based on sliding mode controller only. This simulation analysis builds up the foundation for the next experiments. In the future, it is really necessary to research the influence of the network parameters to the control effects. At the same time, it is also essential to study the active rehabilitation training algorithms to improve the participations of the patients so as to obtain a better rehabilitation training effect.

\section{References}

1. Bernhardt M, Frey M, Colombo G and Riener R 2005 9th International Conference on Rehabilitation Robotics p 536-39

2. Jezernik S, Colombo G and Morari M 2004 IEEE Transactions on Robotics and Automation 20 574-82

3. Riener R, Lunenburger L, Jezernik S, Anderschitz M, Colombo G and Dietz V 2005 IEEE transactions on neural systems and rehabilitation engineering $\mathbf{1 3}$ 380-394

4. $\mathrm{Lu} \mathrm{R}$, Li Z, Su C-Y and Xue A 2014 IEEE Transactions on Industrial Electronics 61 3776-3785

5. del-Ama AJ, Gil-Agudo Á, Pons JL and Moreno JC 2014 Journal of neuroengineering and rehabilitation 1127

6. Suzuki K, Mito G, Kawamoto H, Hasegawa Y and Sankai Y 2007 Advanced Robotics 21 1441-1469

7. Puentes S, Kadone H, Kubota S, Abe T, Shimizu Y and Marushima A 2018 Frontiers in neuroscience 12 99

8. He Y, Eguren D, Azorín JM, Grossman RG, Luu TP and Contreras-Vidal JL 2018 Journal of neural engineering 151004

9. Chen B, Zhong C-H, Zhao X, Ma H, Guan X and Li X 2017 Journal of orthopaedic translation 11 7-18.

10. Pan D, Gao F, Miao Y and Cao R 2015 Advances in Engineering Software 79 36-46

11. Kazerooni H, Racine J-L, Huang L and Steger R 2005 Proceedings of the 2005 IEEE international conference on robotics and automation p 4353-60
12. Li F, Wu Z and Qian J 2013 Sensors \& Transducers 161192

13. Park J and Sandberg IW 1991 Neural computation 3 246-57 